\title{
Analysis of the effect of fingertip size for sex differences in current perception thresholds with transcutaneous electrical stimulation in healthy subjects
}

Shin-ichiro Seno ( $\square$ seno@ks.kyorin-u.ac.jp )

Kyorin University: Kyorin Daigaku

Hideaki Shimazu

Hokuriku University

Eiki Kogure

Kyorin University: Kyorin Daigaku

Atsushi Watanabe

Kyorin University: Kyorin Daigaku

Hiroko Kobayashi

Kyorin University: Kyorin Daigaku

\section{Research note}

Keywords: current perception threshold (CPT), sex differences, electrical stimulation, PainVision®

Posted Date: March 18th, 2021

DOI: https://doi.org/10.21203/rs.3.rs-322314/v1

License: (c) (i) This work is licensed under a Creative Commons Attribution 4.0 International License.

Read Full License 


\section{Abstract \\ Objective}

This study aimed to measure the current perception threshold (CPT) of five fingertips of the left hand in healthy subjects and analyze whether sex differences in perception thresholds are suppressed when adjusting for fingertip size among males and females.

\section{Results}

For fingertips from the thumb to the little finger, the males' CPT values were $1.03,0.83,0.86,0.86$, and $0.88 \mathrm{~mA}$; the females' results were $0.63,0.55,0.54,0.51$, and $0.50 \mathrm{~mA}$. The CPTs were higher in males than in females for every fingertip. Upon adjusting for fingertip length, the log-transformed CPT values were found to have sex differences, except for the index finger: thumb, $t(20.05)=3.493, p=0.002$; middle finger, $U(30)=44.50, p=0.005$; ring finger, $t(30)=55.50, p=0.018$; little finger, $U(30)=30.00, p=0.001$. Similarly, the CPT values, transformed into log values when adjusting for the fingertip area, were found to have sex differences for three fingertips: thumb, $t(18)=2.649, p=0.016$; middle finger, $U(20)=12.00, p=$ 0.004 ; ring finger, $t(18)=2.206, p=0.041$. According to this study, sex differences in CPTs were not completely abolished by adjusting for fingertip length or area.

\section{Introduction}

Perceptive sensitivity to transcutaneous electrical stimulation is termed as the current perception threshold (CPT), and it is used as a method for assessing peripheral nerve function by both researchers and clinicians. CPT measurement is noninvasive, easy, and semi-objective; previous studies have shown that sensitivity to electrical stimulation depends on several physiological parameters, such as sex, age, physical sites, and presence of neuropathy [1-6].

Past studies have pointed to factors that affect sex differences in CPT, including skinfold thickness or quantity of fat tissue [6-8]. Our research group investigated this relationship with CPT and body fat percentage (or body water percentage) and reported an adjustment method to suppress sex differences in sensory sensitivity for electrical stimulation [9]. However, we have observed sex differences in CPTs on the area of the gum, which includes little fat tissue [10].

Existing literature suggests that CPT will also be affected by other parameters, except for body fat percentage or body water percentage. We assume that the size of the measured part may be a factor affecting perceptive sensitivity to transcutaneous electrical stimulation. To test our hypothesis, we measured the CPT at the five fingertips of healthy subjects' left-hand and analyzed whether sex differences in the perception threshold are suppressed when adjusting for fingertip size (length or area) among males and females. 


\section{Materials And Methods}

This study included 62 healthy student volunteers (31 males and 31 females aged 20-23 years). Written informed consent to participate in the study was obtained from all participants. Participants had no visible skin disease and no known history of neurological or psychological disorders. The ethical committee of Kyorin University approved of the study in advance (Approval number; 27 - 7).

To measure CPT on the fingertips, we used PainVision ${ }^{\circledR}$ (PS-2100, Nipro Corporation, Osaka, Japan) [1, 3, 9-12] and a dedicated stimulating electrode (width: $25 \mathrm{~mm}$; depth: $16 \mathrm{~mm}$ ). Two electrode elements of 3$\mathrm{mm}$ diameter were located at the center of the plate with 10-mm intervals between the inside edges of the positive pole and negative pole. The form pat, an EL-PATCH (Nipro Corporation, Osaka, Japan) consisting of a conductive gel and an adhesive tape, was directly attached to the face of the electrode.

Information from participants, including sex, age, and the size of the left hand's five fingertips, such as the length and width from the fingertips to the first joint, were recorded before the CPT measurements. After the stimulation site was sterilized using an alcohol-covered cotton swab and the stimulating electrode was attached, the amplitude of the current was automatically increased to approximately $2.1 \mu \mathrm{A} / \mathrm{s}$ until the participant pushed a stop switch upon first perceiving the electrical stimulation. CPT was determined, and represented by the mean of three measurements, for each fingertip. Throughout the experiment, we assessed the fingertips, in order from their thumb to the little finger, of each participant. The PainVision ${ }^{\circledR}$ outline has been previously described [9].

\section{Statistical analysis}

Shapiro-Wilk test was used to assess the normal distribution of data. If the data were normally distributed, sex differences were assessed with a Student's t-test (homogeneity of variances) or Welch's ttest (non-homogeneity of variances). If the data were not normally distributed, a Mann-Whitney $U$ test was used to assess the measurement values.

Spearman's correlations were performed to determine the relationship between the CPT and the fingertip characteristics (length or area).

The software package SPSS Statistics version 26.0 for Windows (IBM Inc., Armonk, NY, USA) was used for all statistical analyses. $P$ values under .05 were considered statistically significant.

\section{Results}

As shown in Table 1, we observed sex differences in the left-hand fingertip features. 
Table 1

Comparison of the fingertip characteristics in 62 healthy subjects according to sex

\begin{tabular}{|c|c|c|c|c|c|}
\hline & & \multicolumn{2}{|l|}{ length [cm] } & \multicolumn{2}{|l|}{ area $\left[\mathrm{cm}^{2}\right]$} \\
\hline & & mean (SD) & sex differences & mean $(S D)$ & sex differences \\
\hline \multirow[t]{2}{*}{ thumb } & $M$ & $3.17(0.23)$ & $p<0.001$ & $6.40(0.78)$ & $p<0.001$ \\
\hline & $\mathrm{F}$ & $2.84(0.16)$ & $(t=6.42)$ & $5.01(0.53)$ & $(U=67.00)$ \\
\hline \multirow[t]{2}{*}{ index finger } & M & $2.63(0.17)$ & $p<0.001$ & $4.17(0.51)$ & $p<0.001$ \\
\hline & $\mathrm{F}$ & $2.38(0.15)$ & $(t=6.01)$ & $3.38(0.32)$ & $(t=7.38)$ \\
\hline \multirow[t]{2}{*}{ middle finger } & M & $2.74(0.16)$ & $p<0.001$ & $4.41(0.51)$ & $p<0.001$ \\
\hline & $F$ & $2.46(0.15)$ & $(t=7.17)$ & $3.49(0.35)$ & $(t=8.22)$ \\
\hline \multirow[t]{2}{*}{ ring finger } & M & $2.70(0.18)$ & $p<0.001$ & $4.03(0.49)$ & $p<0.001$ \\
\hline & $F$ & $2.43(0.17)$ & $(U=123.00)$ & $3.25(0.39)$ & $(t=6.95)$ \\
\hline \multirow[t]{2}{*}{ little finger } & M & $2.47(0.17)$ & $p<0.001$ & $3.40(0.39)$ & $p<0.001$ \\
\hline & $\mathrm{F}$ & $2.19(0.19)$ & $(t=6.32)$ & $2.66(0.37)$ & $(t=7.60)$ \\
\hline
\end{tabular}

Figure 1(a) represents the distribution of CPT values obtained from each left fingertip according to sex. As a result of having transformed these CPTs into log values, the males' log-transformation values for fingertips from the thumb to the little finger were $-0.02,-0.12,-0.10,-0.11$, and -0.12 ; the females' results were $-0.21,-0.28,-0.28,-0.30$, and -0.31 . The log-transformation values showed sex differences among all fingers: thumb: $t(45.02)=5.82, p<0.001$; index finger: $t(48.93)=3.91, p<0.001$; middle finger: $U(62)=196.50, p<0.001$; ring finger: $U(62)=155.50, p<0.001$; little finger: $U(62)=169.00$, $p<0.001$. Figure 1 (b) shows the relationship between the CPT values and the fingertip characteristics for all participants. As a result of having analyzed the data using Spearman's correlations, a statistically positive correlation was observed between the CPT values and fingertip length $(\rho=0.44, p<0.001)$. Similarly, the relation between the CPT values and the fingertip area was found to have a statistically positive correlation, and the Spearman's correlation coefficient $(\rho)$ was $0.47(p<0.001)$.

Figure 2(a) represents the CPT value results from 15 males with short fingertips and 15 females with long fingertips. The difference in average fingertip length between the two groups was not statistically significant: thumb, $U(30)=98.00, p=0.548$; index finger, $U(30)=107.50, p=0.836$; middle finger, $U(30)=$ 76.00, $p=0.130$; ring finger, $t(28)=0.052, p=0.959$; little finger, $U(30)=76.00, p=0.130$. Despite adjusting for fingertip length, we observed sex differences between the two groups as shown in the log-transformed adjusted CPT values: thumb, $t(20.05)=3.493, p=0.002$; middle finger, $U(30)=44.50, p=0.005$; ring finger, $t(30)=55.50, p=0.018$; little finger, $U(30)=30.00, p=0.001$. There was no significant difference between the groups for the index finger $(t(21.47)=1.96, p=0.063)$. Figure $2(b)$ shows the CPT value results from 
10 males with each small fingertip area and 10 females with each large fingertip area. The difference in average fingertip area between the two groups was not statistically significant: thumb: $U(20)=39.00, p=$ 0.406; index finger: $U(20)=39.00, p=0.406$; middle finger: $U(20)=35.00, p=0.257$; ring finger: $U(20)=$ 40.00, $p=0.450$; little finger: $U(20)=44.00, p=0.650$. Despite adjusting for fingertip area, we observed sex differences in three fingertips between the two groups as shown in the log-transformed adjusted CPT values: thumb, $t(18)=2.649, p=0.016$; middle finger, $U(20)=12.00, p=0.004$; ring finger, $t(18)=2.206, p=$ 0.041 .

\section{Discussion}

The aim of this study was to analyze whether the geometric size of the measurement site which attaches the electrode affects perceptive sensitivity to transcutaneous electrical stimulation, particularly in terms of sex differences in CPT. The data obtained demonstrated significant sex differences in 62 subjects. The CPT value in the thumb was higher than that in any other fingers, regardless of sex, and the CPT values in males were higher than those in females in every fingertip. Moreover, the CPT and finger features (length or area) were found to have a statistically positive correlation.

On the basis of the experimental results, we analyzed whether sex differences in perception threshold are suppressed using two parameters (length and area in the fingertips). As shown in Table 1, two parameters were used to observe sex differences. We selectively chose more than 10 subjects to adjust for sex differences in these parameters. In the case of fingertip length, the selected subjects consisted of 15 males with a short fingertip and 15 females with a long fingertip. In the case of the fingertip area, the selected subjects were 10 males with a small fingertip area and 10 females with a large fingertip area. By analyzing the log-transformation values in these groups, we investigated the possibility of suppressing sex differences in perception sensitivity.

Although we had adjusted for the length or area in each fingertip within the two groups and analyzed the CPT values, the adjusted CPT values were still found to have sex differences, except for some fingertips, as shown in Fig. 2. As such, we have reconsidered our hypothesis and discussed several potential underlying reasons. First, it is suggested that the difference in body fat percentage or body water percentage between sexes may have influenced CPT. The stimulating electric current flows toward a cathode from an anode through three layers: the epidermis, dermis, and subcutaneous tissue. Because electrical conductivity in the fat tissue is lower than that in muscle or blood [13], most electrical currents may flow easily through the epidermis and dermis. As a result, females with a higher fat mass had epidermal nerve fibers that were more easily excited than those of participants whose body fat percentage was low, resulting in a decreased perception threshold. In fact, we found that CPT values in males were changed by fat percentage [9]. Second, it is likely that the perceptive sensitivity to electrical stimulation was affected by skin thickness. It has been reported that skin thickness in various body parts reflected sex differences [14] and that the cornified layer in fingertips was significantly thinner in females than in males [15]. The corneum, when dry, acts as an impedance to the stimulating current [16]. Therefore, it may have been necessary to investigate not only visibility geometric information regarding 
the fingertips but also the skin thickness. Third, we may consider that individual sensitivity to electrical stimulation, and sex differences of CPT, may be influenced by the number of epidermal nerve fibers. The density of intra-epidermal nerve fibers (IENFs) is low in elderly people $[17,18]$ and patients with diabetes mellitus $[19,20]$. Furthermore, CPT values in these groups are higher than those in healthy subjects $[1,2$, 5]. Similarly, females are reported to have more IENFs than males do [21, 22]. Thus, sex differences in CPT values could be explained by the distribution density of IENFs. In fact, differences in IENF quantity are posited to alter the thresholds of warmth and cold perception [23]. Taken together, the number of IENFs under the stimulating electrode must play a key role in sensitivity to electrical stimulation.

Moreover, it has been recently reported that the nerve conducting pathway in the central nervous system is different between the two sexes [24]. For this reason, it is likely that the sex difference in pain perception is caused by differences in the nerve conducting pathway. This finding may be supported by the fact that CPT is different between males and females.

\section{Limitations}

We recognize that this study has a few limitations. First, subjects consisted of 62 healthy volunteer college students in their twenties from Kyorin University. However, the adjusted data were limited to 1015 subjects, such that results could potentially differ when a greater number of subjects are tested. Second, information on subjects only included sex, age, and fingertip size in this study. Although the CPTs in some fingertips abolished sex differences by adjusting for finger length or area, we could not completely achieve the goal of this study. Thus, we should analyze CPT while taking into account the characteristics of each body part, such as subcutaneous fat volume, body water percentage, and skin thickness. Third, we must revalidate the method of measurement of perception threshold using PainVision ${ }^{\circledR}$. This device measures CPT by automatically increasing the amplitude of the current. Because the CPT values in males were significantly different than those of females ( $F$-test: thumb, $p<$ 0.001 ; index finger, $p=0.003$; middle finger, $p=0.002$; ring finger, $p<0.001$; little finger, $p<0.001$ ), this problem may be solved by using waxing and waning electrical stimulation. In addition, the CPTs obtained using PainVision ${ }^{\circledR}$ were also likely under the influence of central sensitization in electrical stimulation. Thus, the evaluation of the CPT in clinical fields is not only an evaluation of the peripheral nerve function but should also be considered with regard to various influences.

\section{Abbreviations}

CPT: current perception threshold; IENF: intra-epidermal nerve fiber

\section{Declarations}

\section{Ethics approval and consent to participate}

Written informed consent to participate in the study was obtained from all subjects. The ethical committee of Kyorin University approved the study in advance (Approval number; 27-7). 


\section{Consent for publication}

Not applicable.

\section{Availability of data and materials}

The data in this study are not publicly available because the participants were promised that the raw data would remain confidential. However, they are available from the corresponding author on reasonable request.

\section{Competing interests}

The authors declare that they have no competing interests.

\section{Funding}

No external funding was available for this study, except for the support of Kyorin University.

\section{Authors' contributions}

SS planned the study concepts and design, conducted the experiments, and analyzed the data. EK, AW, and HK contributed to the critical revision of the manuscript. HS provided important intellectual input to complete this manuscript. All authors approved the final version of this manuscript.

\section{Acknowledgements}

Not applicable.

\section{References}

1. Seno S, Kato S, Kobayashi H, Watanabe A, Shimazu H. Evaluation of sex and age differences in the perception threshold of body surface against electrical stimulation: measurement of perception threshold to determine the possibility of diabetic neuropathy diagnoses. Trans JSMBE. 2011; https://doi.org/10.11239/jsmbe.49.163.

2. Oliveira FB, Fachin-Martins E, Couto-Paz C, Martins HR, Tierra-Criollo CJ, Coste CA. Current perception threshold through sinusoidal electrical stimulation at different frequencies in a comparative assessment for subjects affected and non-affected by diabetes mellitus. IEEE Xplore. 2015; https://doi.org/10.1109/NER.2015.7146772.

3. Nishimura T, Nakae A, Shibata M, Mashimo T, Fujino Y. Age-related and sex-related changes in perfusion index in response to noxious electrical stimulation in healthy subjects. J Pain Res. 2014; http://dx.doi.org/10.2147/JPR.S57140.

4. Fujihara A, Ukimura O, Iwata T, Miki T. Neuroselective measure of the current perception threshold of A-delta and C-fiber afferents in the lower urinary tract. Int J Urol. 2011; 
https://doi.org/10.1111/j.1442-2042.2011.02749.x.

5. Matsutomo R, Takebayashi K, Aso Y. Assessment of peripheral neuropathy using measurement of the current perception threshold with the Neurometer in patients with type 2 diabetes mellitus. $J$ Int Med Res. 2005; https://doi.org/10.1177/147323000503300410.

6. Leong GW, Lauschke J, Rutowski SB, Waite PM. Age, gender, and side differences of cutaneous electrical perceptual threshold testing in an able-bodied population. J Spinal Cord Med. 2010; https://doi.org/10.1080/10790268.2010.11689702.

7. Maffiuletti NA, Herrero AJ, Jubeau M, Impellizzeri FM, Bizzini M. Differences in electrical stimulation thresholds between men and women. Ann Neurol. 2008; DOI: 10.1002/ana.21346.

8. Geng B, Yoshida K, Jensen W. Impacts of selected stimulation patterns on the perception threshold in electrocutaneous stimulation. J Neuroeng Rehabil. 2011; https://doi.org/10.1186/1743-0003-8-9.

9. Seno S, Shimazu H, Kogure E, Watanabe A, Kobayashi H. Factors affecting and adjustments for sex differences in current perception threshold with transcutaneous electrical stimulation in healthy subjects. Neuromodulation. 2018; https://doi.org/10.1111/ner.12889.

10. Seno S. Perception threshold by the electrical stimulation on oral cavity and lip regions. Trans JSMBE. 2011; https://doi.org/10.11239/jsmbe.49.925.

11. Shimazu H, Seno S, Kato S, Kobayashi H, Akimoto M. Development of a quantitative measurement method for the magnitude of pain using painless electrical stimulation and its evaluation using experimental pain. Trans JSMBE. 2005; https://doi.org/10.11239/jsmbe.43.117.

12. Kim J, Lee KS, Kong SW, Kim T, Kim MJ, Park SB, Lee KH. Correlations between electrically quantified pain degree, subjectively assessed visual analogue scale, and the McGill pain questionnaire: a pilot study. Ann Rehabil Med. 2014; https://doi.org/10.5535/arm.2014.38.5.665.

13. Schwan HP. Electrical properties of tissue and cell suspensions. In: Lawrence JH, Tobias CA, editors. Advances in biological and medical physics. Volume 5. Cambridge, MA: Academic Press; 1957. p. 147-209.

14. Waller JM, Maibach HI. Age and skin structure and function, a quantitative approach (I): blood flow, $\mathrm{pH}$, thickness, and ultrasound echogenicity. Skin Res Technol. 2005; https://doi.org/10.1111/j.0909725X.2005.00151.x.

15. Fruhstorfer $H$, Abel $U$, Garthe $C D$, Knuttel A. Thickness of the stratum corneum of the volar fingertips. Clin Anat. 2000; https://doi.org/10.1002/1098-2353(2000)13:6<429::AID-CA6>3.0.C0;2-5.

16. Reilly JP. Electrical stimulation and electropathology. New York: Cambridge University Press; 1992. p. 22-33.

17. McArthur JC, Stocks EA, Hauer P, Cornblath DR, Griffin JW. Epidermal nerve fiber density: normative reference range and diagnostic efficiency. Arch Neurol. 1998; DOI: 10.1001/archneur.55.12.1513.

18. Kelly EJ, Terenghid G, Hazarid A, Wiberg M. Nerve fibre and sensory end organ density in the epidermis and papillary dermis of the human hand. Br J Plast Surg. 2005; DOI:

10.1016/j.bjps.2004.12.017. 
19. Polydefkis M, Hauer P, Sheth S, Sirdofsky M, Griffin JW, McArthur JC. The time course of epidermal nerve fibre regeneration: studies in normal controls and in people with diabetes, with and without neuropathy. Brain. 2004; DOI: 10.1093/brain/awh175.

20. Sorensen L, Molyneaux L, Yue DK. The relationship among pain, sensory loss, and small nerve fibers in diabetes. Diabetes Care. 2006; DOI: 10.2337/diacare.29.04.06.dc05-2180.

21. Gøransson LG, Mellgren SI, Lindal S, Omdal R. The effect of age and gender on epidermal nerve fiber density. Neurology. 2004; https://doi.org/10.1212/01.WNL.0000113732.41127.8F.

22. Lauria G, Bakkers M, Schmitz C, Lombardi R, Penza P, Devigili G, Smith A G, Hsieh ST, Mellgren SI, Umapathi T, Ziegler D, Faber CG, Merkies IS. Intraepidermal nerve fiber density at the distal leg: a worldwide normative reference study. J Peripher Nerv Syst. 2010; DOI: 10.1111/j.15298027.2010.00271.x.

23. Løseth S, Lindal S, Stalberg E, Mellgren I. Intraepidermal nerve fibre density, quantitative sensory testing and nerve conduction studies in a patient material with symptoms and signs of sensory polyneuropathy. Eur J Neurol. 2006; DOI: 10.1111/j.1468-1331.2006.01232.x.

24. Dance A. Why the sexes don't feel pain the same way. Nature. 2019; doi: 10.1038/d41586-01900895-3.

\section{Figures}

a.

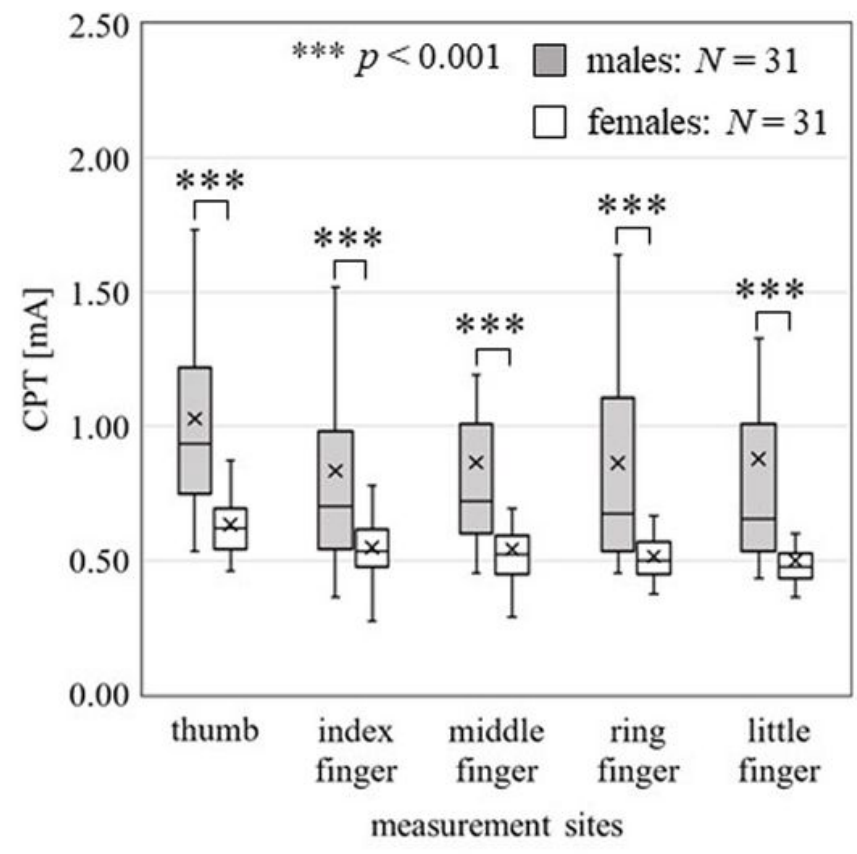

b.

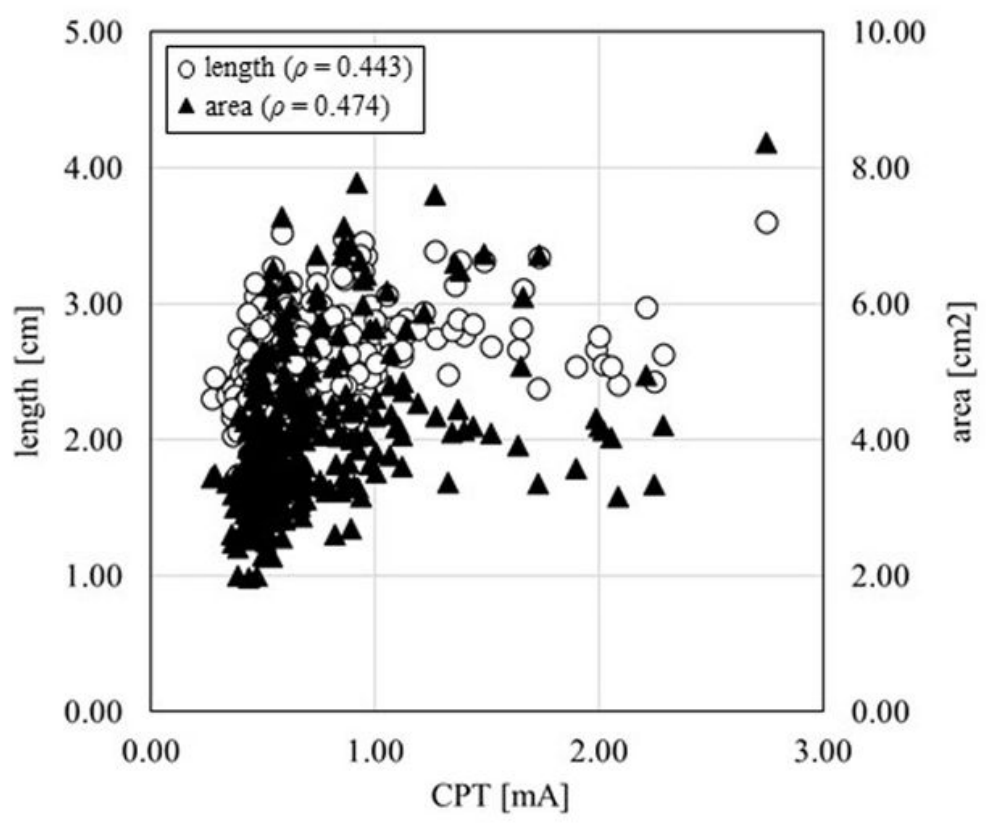

\section{Figure 1}

Relationship between CPT data and fingertip characteristics (a) Comparison of CPTs among the fingertips of the left hand in male $(\mathrm{N}=31)$ and female $(\mathrm{N}=31)$ subjects. Data are shown for each fingertip 
and expressed as box plots for males (gray) and females (white). The box limits are the 25th and 75th percentiles. The whiskers indicate the minimum and maximum values. The crosses denote the mean. The horizontal lines are the median values. The asterisks denote a significant difference between the sexes ( $* \star \star ~ p<0.001)$. A comparison of the CPT values within both the male and female groups shows statistically significant differences across the five fingertips (Friedman test: $p<0.001$, not shown). (b) Relationship between the CPT data and fingertip characteristics (length or area) for all participants. The data are expressed as a scatter diagram consisting of the aspects of both length $(\mathbf{O})$ or area $(\boldsymbol{\Lambda})$. As a result of having analyzed the data using Spearman's correlations, the CPT values and fingertip length were positively correlated $(\rho=0.44, p<0.001)$. Similarly, the CPT values and fingertip area were also positively correlated $(\rho=0.47, p<0.001)$.

a. length

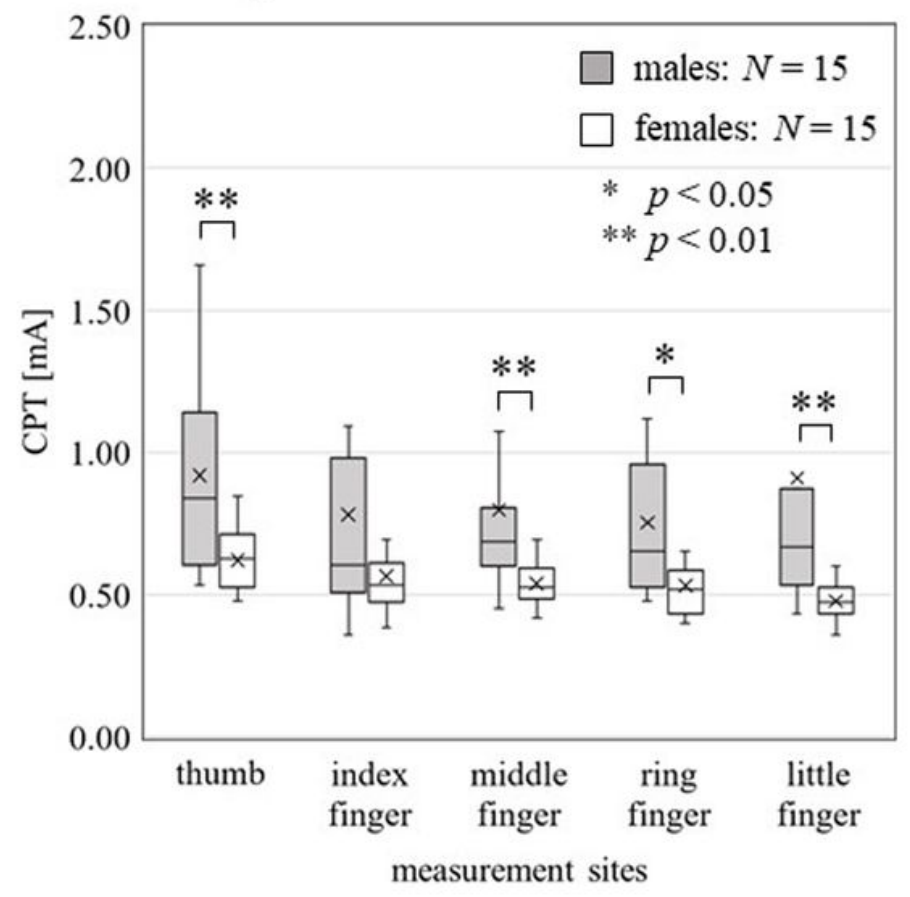

\section{b. area}

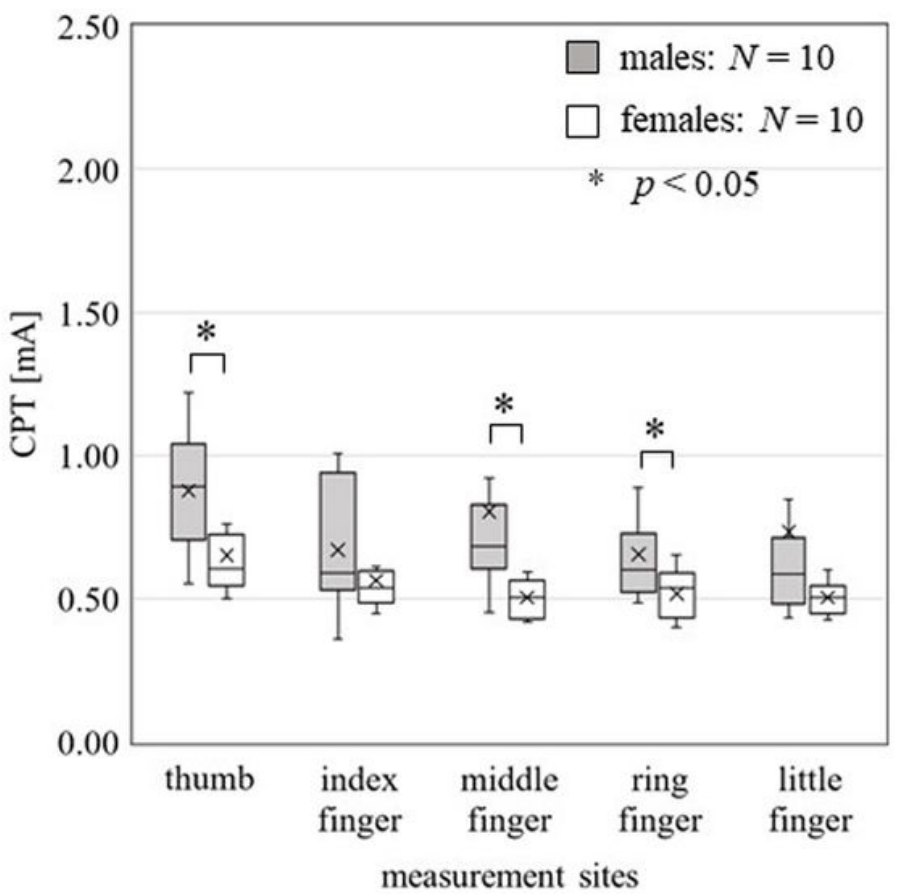

\section{Figure 2}

CPT comparison adjusting for the fingertip's length or area between the two sexes Data are shown for each electrode and expressed as box plots for males (gray) and females (white). The box limits are the 25th and 75th percentiles. The whiskers indicate the minimum and maximum values. The crosses denote the mean. The horizontal lines are the median values. The asterisks denote a significant difference between the sexes $\left(* \star p<0.01,{ }^{*} p<0.05\right)$. (a) Depicts the data measured from 15 males and 15 females with similar fingertip lengths (thumb: male, $2.97 \mathrm{~cm}$; female, $2.97 \mathrm{~cm} ; \mathrm{p}=0.55$; index finger: male, $2.50 \mathrm{~cm}$; female, $2.51 \mathrm{~cm} ; p=0.84$; middle finger: male, $2.62 \mathrm{~cm}$; female, $2.58 \mathrm{~cm} ; \mathrm{p}=0.13$; ring finger: male, $2.57 \mathrm{~cm}$; female, $2.56 \mathrm{~cm} ; \mathrm{p}=0.13$; little finger: male, $2.33 \mathrm{~cm}$; female, $2.33 \mathrm{~cm} ; \mathrm{p}=0.15)$. After having transformed CPT values into the log values, each log value was found to have sex differences: thumb: $t(20.05)=3.493$, $p=0.002$; middle finger: $U(30)=44.50, p=0.005$; ring finger: $t(30)=55.50, p=0.018$; little finger: $U(30)=30.00$, $p=0.001$, except for index finger $(t(21.47)=1.96, p=0.063)$. (b) Shows the data measured from 10 males 
and 10 females with similar fingertip areas (thumb: male, $5.61 \mathrm{~cm} 2$; female, $5.61 \mathrm{~cm} 2 ; \mathrm{p}=0.41$; index finger: male, $3.64 \mathrm{~cm} 2$; female, $3.73 \mathrm{~cm} 2 ; p=0.41$; middle finger: male, $3.91 \mathrm{~cm} 2$; female, $3.88 \mathrm{~cm} 2$; $p=0.26$; ring finger: male, $3.53 \mathrm{~cm} 2$; female, $3.66 \mathrm{~cm} 2 ; p=0.45$; little finger: male, $2.99 \mathrm{~cm} 2$; female, 3.05 $\mathrm{cm} 2 ; \mathrm{p}=0.65)$. After having transformed CPT values into the log values, each log value was found to have sex differences: thumb: $\mathrm{t}(18)=2.649, \mathrm{p}=0.016$; middle finger: $\mathrm{U}(20)=12.00, \mathrm{p}=0.004$; ring finger:

$t(18)=2.206, p=0.041$, except for index finger $(t(18)=1.174, p=0.256)$ and little finger $(U(20)=27.50$, $\mathrm{p}=0.088)$. 